\title{
BESIII Simulation Software
}

\author{
Ziyan Deng ${ }^{1}$ (dengzy@ihep.ac.cn)
}

Huaimin Liu (liuhm@ihep.ac.cn)

Guofu Cao (caogf@ihep.ac.cn)

Miao He (hem@ihep.ac.cn)

Ye Yuan (Yuany@ihep.ac.cn)

Institute of High Energy Physics

Yuquanlu 19(2), Beijing 100049, P. R. China

Zhengyun You (youzy@hep.pku.edu.cn)

Yutie Liang (liangyt@hep.pku.edu.cn)

Peking University

Beijing 100871, P. R. China

The BESIII is a general-purpose experiment for studying electron-positron collisions at BEPCII, which is currently under construction at IHEP, Beijing. The BESIII offline software system (BOSS) is built on the Gaudi architecture. The Simulation software is developed in BOSS framework. This contribution introduces the implementation of simulation algorithm for BESIII detector, and it's performance.

XI International Workshop on Advanced Computing and Analysis Techniques in Physics Research Amsterdam, the Netherlands

23-27 April, 2007

\footnotetext{
$1 \quad$ Speaker
} 


\section{Introduction}

The Beijing Electron Positron Collider II (BEPCII), the upgrade of BEPC, is a multi-bunch $\mathrm{e}+\mathrm{e}-$ collider that is scheduled to provide collision in 2008. The BESIII[1] is a general-purpose detector being built to meet requirements of physics studies at BEPCII. The BESIII detector consists of the flowing components:

(1) A He gas based Main Drift Chamber (MDC) with a single wire resolution better than $130 \mu \mathrm{m}$.

(2) A CsI Electromagnetic Calorimeter (EMC) with an energy resolution better than 2.5\% at $1 \mathrm{GeV}$.

(3) A Time-of-Flight (TOF) system with a time resolution better than 100ps.

(4) A super-conducting solenoid magnet with a field of 1.0 Tesla.

(5) A RPC based Muon Chamber (MUC) system.

The BESIII Offline Software System (BOSS) is developed using C++ language and objectoriented techniques on the operation system of Scientific Linux CERN (SLC3). The CMT[2] is used as a software configuration tool. The BOSS framework has been developed based on Gaudi[3], which provides standard interfaces for the common software components necessary for data processing and analysis. The whole data processing and physics analysis software consists of five functional parts: simulation, calibration, reconstruction, analysis tools and event display. Data flow in BOSS framework in shown in Figure 1. Algorithms can retrieve data objects from transient data store, and also register new data objects to TDS.

The simulation algorithm in BOSS is developed based on Geant4 toolkit, using lots of external HEP libraries including CERNLIB, CLHEP, ROOT[4], GDML[5], Xerces_c etc. The whole simulation software consists of the following components: detector description, event generator, physics processes, magnetic field, digitization, MC truth, trigger simulation and background mixing.

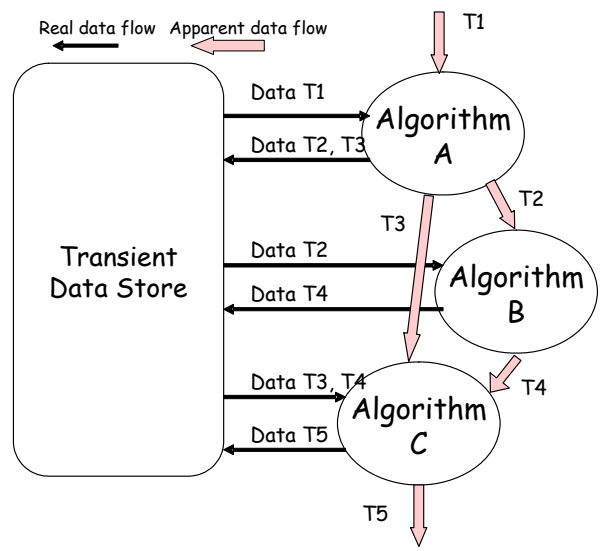

Figure1: Data flow in BOSS framework

\subsection{Detector Description}

The detector's material and geometry needed for detector simulation are constructed with Geant 4 classes. Due to complicated structure, the standard shapes in Geant4 are not general enough to describe all BESIII sub-detector modules. For example, the G4TwistedTube[6] shape is used to describe MDC stereo cells (shown in Figure 2), and G4IrregularBox[7] shape is used 
for EMC endcap(shown in Figure 3). After importing these two external packages, the BESIII detector can be successfully constructed.

The material and geometry constructed with G4 classes can be written out into GDML data files. After expanding the Geant4 schema and developing a new ROOT schema, the GDML data files can be used in simulation, reconstruction, track extrapolation, and event display. Thus the description of BESIII detector in the whole offline software system is unique.

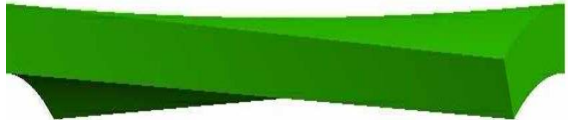

Figure 2: MDC stereo cell

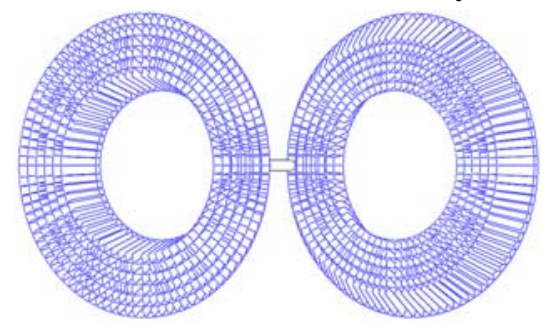

Figure 3: EMC end-cap

\subsection{Event Generator}

The BESII experiment had about 30 generators written in Fortran language that become BESIII's legacy. In order to integrate the legacy generators with the BOSS framework, we use $\mathrm{C}++$ HepEvt_Wrapper class to access the kinematics information of the generated particles, then create a HepMC object containing the kinematics information, and finally register it in Transient Data Store through the Event Data Service. A downstream algorithm retrieves the HepMC object from TDS and use G4HepMCInterface to convert the HepMC object to G4Event for G4 tracking. A physics event generated by BESII generator and tracked in BESIII detector is shown in Figure 4.

Besides the BESII generators, some new generators have been developed for BESIII, such as EvtGen, Bhlumi, KKMC.

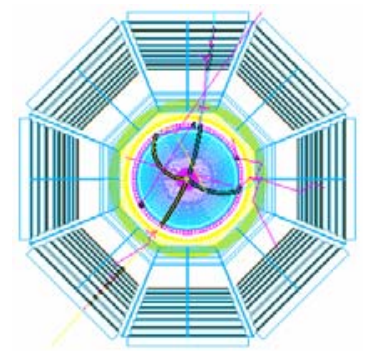

Figure 4: $\psi^{\prime} \rightarrow \pi^{+} \pi^{-} J / \psi \rightarrow \pi^{+} \pi^{-} \mu^{+} \mu^{-}$

\subsection{Physics Processes}

Different hadronic models are provided in Geant4, such as LEP/HEP parameterized model (LHEP), and Quark-Gluon String Precompound model (QGSP), etc. Decay, transportation and standard electromagnetic processes are included in these models. According to the test in our simulation algorithm, there's trivial difference between standard and low energy EM processes. Since Optical photon processes occupy too much time, it can't be used in our simulation algorithm. 
During our test with Geant4.8.1+p01 in large sample, dead-loop and crash are detected. After careful check with G4 track and step information, we found they are mostly caused by bugs in hadronic processes. We found bugs in the following G4 classes:

G4LEAntiProtonInelastic,

G4HadronElastic,

G4UHadronElasticProcess,

G4NucleiProperties,

G4InelasticInteraction.

About the above classes, we have made modification or protection in our Geant4 package to make our simulation software can run smoothly.

\section{$1.4 \quad$ Magnetic Field}

Currently, 1 Tesla uniform magnetic field is used in simulation and reconstruction algorithm. Besides, a 3D magnetic field service provides the value of magnetic field for each space point within the detector. Then simulation and reconstruction algorithm can use the same 3D magnetic field in BOSS framework. The linear interpolated algorithm has been checked carefully. Figure 5 and 6 show the magnetic field got from Super-conducting magnet group, and the magnetic field in BOSS framework.

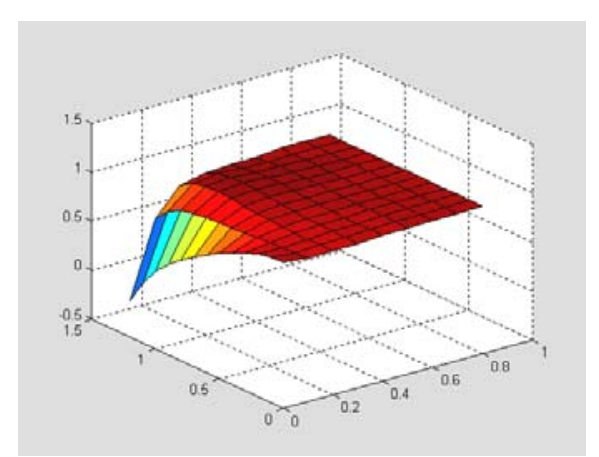

Figure 5: magnetic field from SCM group

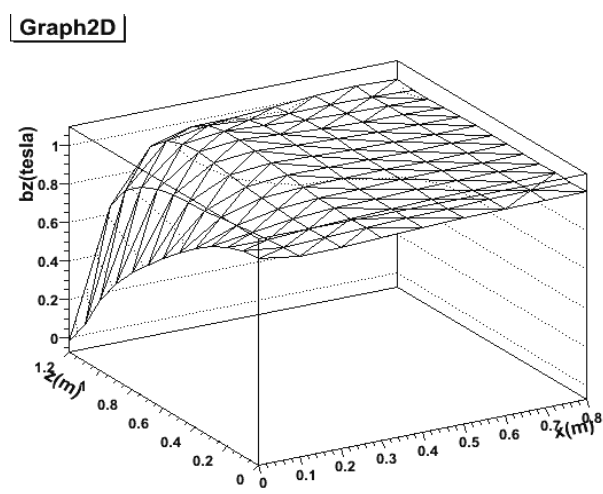

Figure 6: magnetic field in BOSS

\subsection{Digitization}

Digitization is the most important and complicated part of simulation. It's highly depend on detector performance.

For MDC, drift distance is calculated in the hit cell, and using X-T relationship of this cell, drift time is calculated via drift distance. The fast drift time is selected among all the hits in one cell. At the same time, energy deposit in this cell is sum up.Wire resolution and noise also added during digitization.

For TOF, after getting hit information such as energy deposit, time, position etc, light emission and light propagation in scintillator is simulated. Then we get the number of optical photons and the arrival time of each photon. Using the information about optical photons, we simulate the response of photo-multiplier tube to get the signal pulse of PMT, and discriminate the pulse with low level and high level threshold to get TDC and ADC. 
For EMC, waveform method is used to simulate the output of main amplifier, using the output signal, we get the rise time and energy deposit in the crystal. The rise time is provided to eliminate noise.

For MUC, we just get the ID of hit strip, and add noise during digitization.

\subsection{MC truth}

The purpose of MC truth is to provide truth information for reconstruction and physics analysis. We need to save the true state of the particles and their association with detector response. The recorded primary particles include particles from generators and their uninterrupted decayed daughters. The detector response includes the hits in sub-detectors associated with the primary particles.

In order to fully describe the primary particles and their decay chain, we associate each particle with track and vertex object. PDGcode, charge, original and terminal vertex, momentum, decayed daughters are recorded in track object. Parent track, position, time information are recorded in vertex object. Considering the demand of each reconstruction developers, for MDC and MUC, we record the hit history of each primary particle. For TOF, we record the first hit. For EMC, also record the first hit, and at the same time, we sum up the energy loss caused by each primary particle.

\subsection{Trigger Simulation}

Trigger simulation with three sub-detectors has almost completed. Trigger with MUC is ongoing. Trigger algorithm (Figure 7) first get digits of three sub-detectors, and find segment and track in MDC, and clusters in EMC, get energy sum and balance in EMC and hit count in TOF. All the above information is used to match the trigger conditions of each sub-detector.

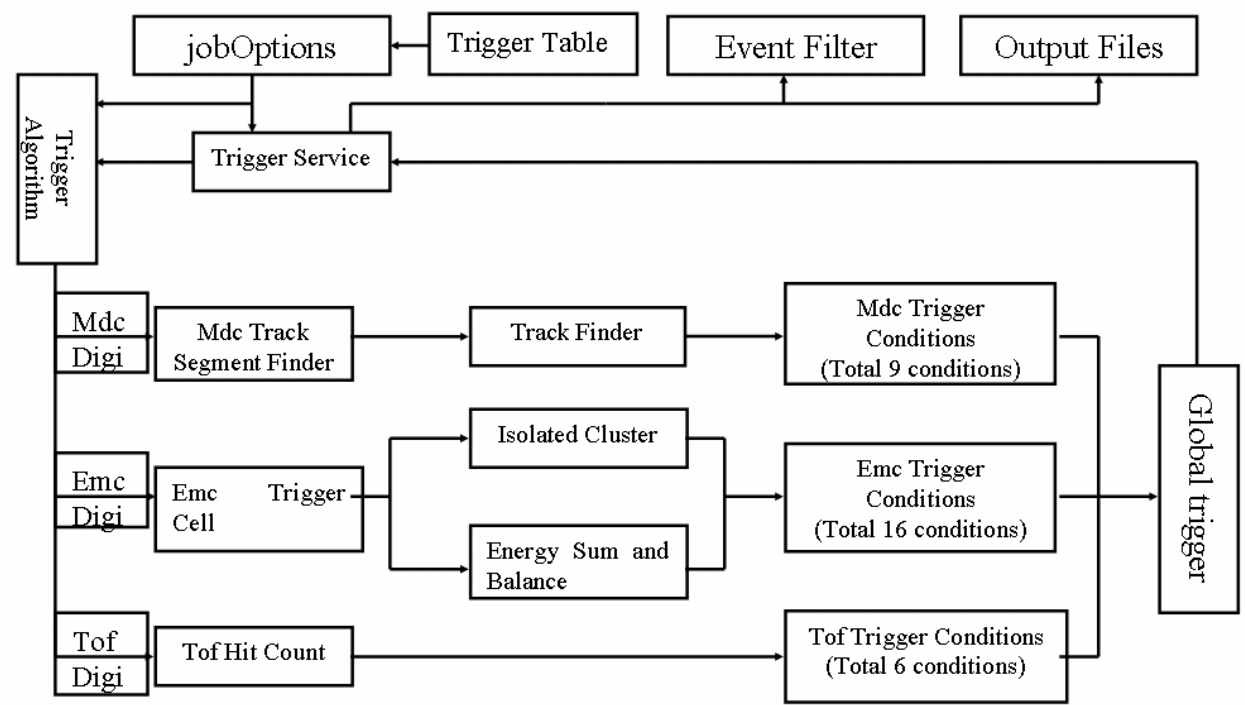

Figure 7: flow chart of trigger simulation

\subsection{Background Mixing}

Event mixing algorithm is developed by Dubna group. This algorithm mixes the signal event with background event in digit level. During the mixing, only signals and noise with the 
same identifier are added up. If no corresponding MC digit found, new digit is created with background information. New charge is sum of MC charge and background charge. New time measurement is the earliest of two. If requested, these rules can be changed individually for each sub-detector.

About the background data, trigger group did some study on simulated background, the MC background events are used in mixing. There were some discussion on background data taking, it may be taken with single beam of separated beam without collision, or taken with random trigger.

\subsection{Performance}

The simulation speed is depend on the event type, the average is 0.9 second per physics event. It's about 1.5 times slower than BESII simulation, which is developed based on G3. About the speed of Geant4, A study group has started to address this issue, and continued with more priority.

For the performance of MDC, the spatial (Figure 8) and momentum resolution (Figure 9) of $\mu^{-}$with $1 \mathrm{GeV} / \mathrm{c}$ transverse momentum is $110 \mu \mathrm{m}$ and $0.4 \%$. This reconstructed result is got based on the digitization which get parameters from MDC beam test.

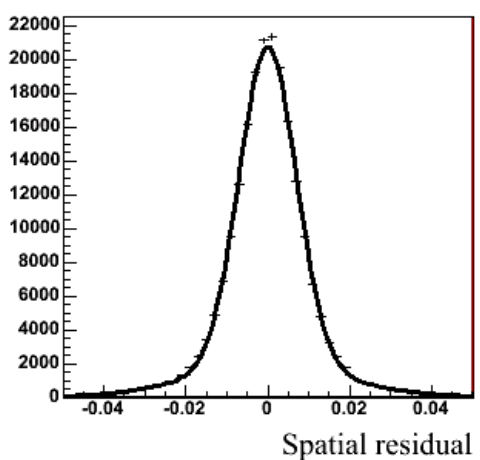

Figure 8: spatial resolution

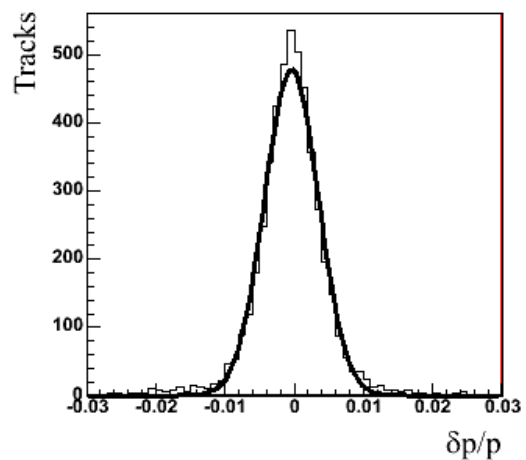

Figure 9: momentum resolution

For TOF, Figure 10 shows the time resolution as a function of $\mathrm{z}$ hit position on barrel TOF scintillator. For $1 \mathrm{GeV} / \mathrm{c}$ electron, at the center of scintillator bar, the time resolution is $85 \mathrm{ps}$. The results from beam test is $80-90$ ps.

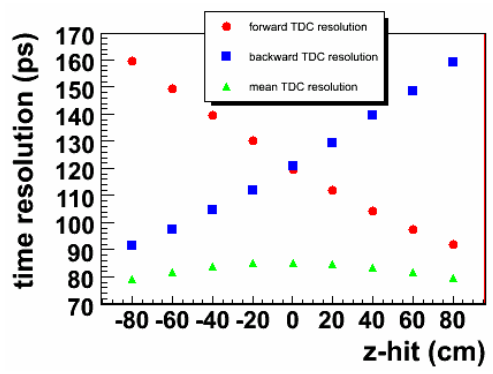

Figure 10: time resolution vs $\mathrm{z}$ hit position

For EMC, Figure 11 shows the energy resolution as a function of the energy of gamma. The resolution of $1 \mathrm{GeV}$ is $2.1 \%$. Figure 12 shows the spatial resolution of gamma at $1 \mathrm{GeV}$, the fit result is $5.8 \mathrm{~mm}$. 


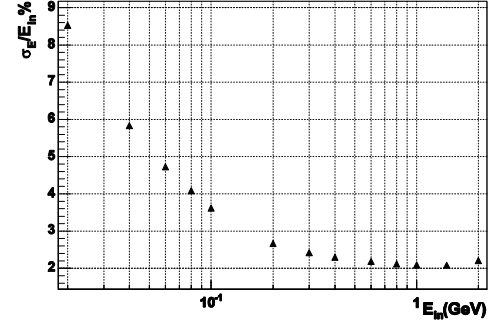

Figure 11: energy resolution vs E

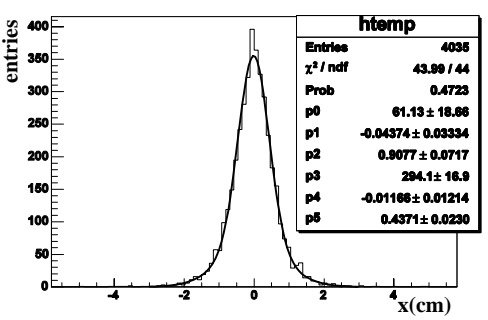

Figure 12: spatial resolution

For MUC, the muon identification efficiency is $95 \%$ at $1 \mathrm{GeV}$, and pion mis_ID probability is $6 \%$. They are all consitent with the designed performance of MUC.

\subsection{Summary}

Simulation software developed in BOSS framework runs stably. Key quantities are consistent with designed target. Mass production has started for physics study. In order to meet the experiment's increasing requirements, the BESIII simulation software may still evolve, more studies on physics processes and digitization are needed. However, the software's performance tuning will become a long-term task.

\section{References}

[1] http://bes3.ihep.ac.cn

[2] C.Arnault, CMT: a Software Configuration Management Tool, Padova, February 2000, Pr

[3] Barrand, G. and others, GAUDI - A software architecture and framework for building HEP data processing applications, Comput.Phys.Commun., 140:45-55,2001

[4] http://root.cern.ch

[5] http://gdml.web.cern.ch/GDML

[6] K. Hoshina et.al., Development of Geant4 Solid for Stereo Minijet cells in a Cylindrical Drift Chamber, Comput.Phys.Commun.153:373-391,2003

[7] http://annwm.lbl.gov/G4/G4.cgi 